\title{
Meeting Summary
}

\section{Urban-Scale Processes in High-Spatial- Resolution Earth System Models}

Ashish Sharma, Donald J. Wuebbles, Rao Kotamarthi, Katherine Calvin, Beth Drewniak, Charles E. Catlett, and Robert Jacob

Workshop on Urban Scale Processes and Their Representation in High Spatial Resolution Earth System Models

What: More than 60 international experts in urban issues and models examined strategies to move forward with better representation of urban processes and environs across local, regional, and global scale models.

When: 22-24 May 2019

Where: Lemont, Illinois 
AfFILIATIONS: Sharma-Illinois State Water Survey, Prairie Research Institute, and Department of Atmospheric Sciences, University of Illinois at Urbana-Champaign, Champaign, Illinois; WuebblesDepartment of Atmospheric Sciences, University of Illinois at Urbana-Champaign, Champaign, Illinois; Kotamarthi, Drewniak, and Jacob-Environmental Science Division, Argonne National Laboratory, Lemont, Illinois; Calvin—Joint Global Change Research Institute, Pacific Northwest National Laboratory, College Park, Maryland; Catlett-Discovery Partners Institute, University of Illinois, and Mansueto Institute for Urban Innovation, University of Chicago, Chicago, Illinois

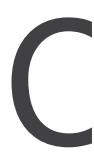

ities and their associated urban areas have a much larger impact on the environment than their spatial footprint on the Earth's surface. This is undoubtedly true at local and regional scales, but also at global scales. At this time, more than half of the world's population lives in urban areas (80\% in the United States), and the global proportion is projected to climb to $70 \%$ by 2050 . Therefore, numerical models to study physics, chemistry, and biology affecting the Earth system at regional and global scales must represent the effects of urban areas on climate and the effects of a changing climate on urban areas. At the same time, it is essential to develop state-of-the-art, simple, and accurate urban models to better understand the relevant processes and also to address issues related to urban security against the spectra of chemical, biological, and radiological (CBR) hazards.

Urban areas have unique landscape characteristics, including mixed land-cover features; built infrastructure for commercial, housing, and transportation; and high-density energy and water consumption. These characteristics determine urban-surface properties and give rise to unique urban climate at micro-, regional, and global scales. Apart from the meteorological interactions, urban environments also face challenges related to potential biochemical, air quality, and fire-safety-related issues. Transport and diffusion processes in urban canyon models are complex due to channeling and turbulent flows and need improved parameterizations (Fernando 2010). Winds may increase/decrease through urban canyons and change weather effects (e.g., wind chill and urban heat island effects), decrease visibility, and adversely affect low-altitude future commercial aerial vehicle operations. Urban inversion layers can adversely affect air quality and urban health. Thus, representing these urban microclimates in climate models is not only important to improve simulations over urban landscapes but is also necessary to improve the climate projections over regions surrounding an urban area.

A 2.5-day international workshop was held at the Argonne National Laboratory (ANL) in the Chicago area in spring 2019. This workshop brought together more than 60 national and international experts to develop a roadmap for a better understanding of the issues associated with urban areas and at enhancing the capabilities of regional and global Earth system models (ESMs) in representing the atmospheric dynamics and chemistry, unique aspects of the biosphere and land use, and human dimensions of the urban environment (Fig. 1). The workshop goal was structured around these questions:

1) What are the current capabilities and gaps in handling urban representation in ESMs? Why is urban representation in an ESM important? What is known? What is important to represent in ESMs-what is included now, and what is not included? How does urban representation change with increase in resolution of the ESMs?

2) What is the current state of urban-scale datasets, including in situ and satellite observations, and the emerging smart city sensing technologies and their possible use in model development including high-resolution land use and ecological datasets?

3) What are the next steps to get a better representation of urban systems in ESMs across scales, and what can the scientific community do to achieve them? 


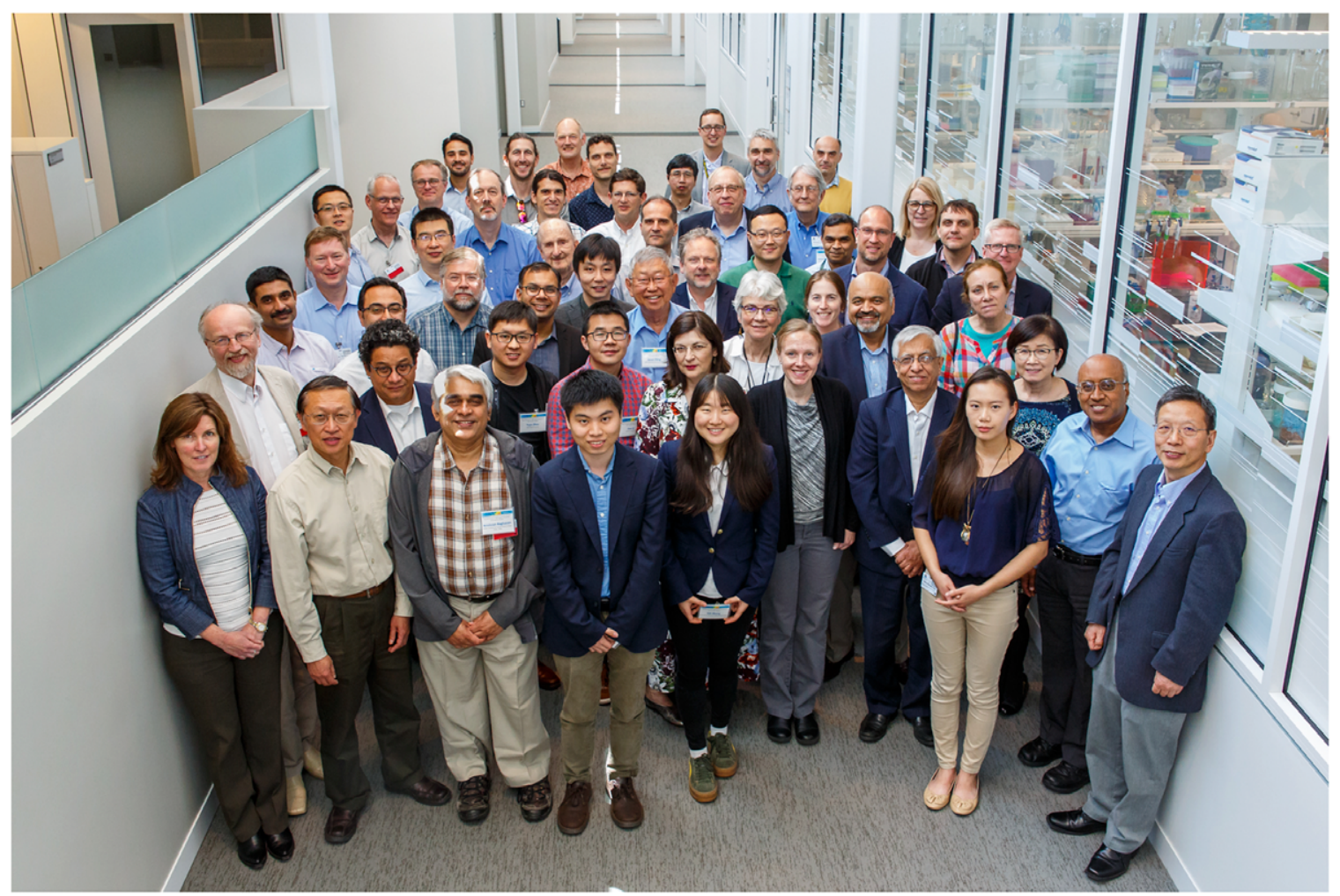

Fig. 1. Photo of the workshop participants from 23 May 2019 (some attendees are missing). There are also reflections affecting the quality of the picture.

This workshop was timely in that the science community is actively developing very highresolution versions of the global ESMs and enhancing ESM capabilities for representing the atmospheric dynamics and chemistry, unique aspects of the biosphere and land use, and human dimensions at the urban scale. This workshop was especially important to those developing very high-resolution versions of regional and global models to improve our understanding of urban physical sciences (vis-à-vis meteorology, climatology, air quality, and observations), and integrating physical sciences with social sciences, energy, and human health across space-time scales.

The workshop began with short, provocative keynote talks on the importance of enhanced representation of urban processes in ESMs, what is known, what is not included in current ESMs, and how ESMs are evolving as we go to higher resolution. The keynote talks opened a Pandora's box of discussions that turned out to be healthy and intense brainstorming during panel and breakout sessions throughout the workshop. Participants listened to multiple federal and international agencies' current and future research directions. Most of the workshop was divided into breakout sessions ranging from (i) challenges of modeling urban systems in ESMs, (ii) modeling of urban processes and their treatment in ESMs, (iii) urban models based on the first principles of computational fluid dynamics (CFD), (iv) urban-scale datasets including ecological and other datasets, (v) emerging sensing technologies and their use, and (vi) the need for ultra-high-resolution land use datasets. During the breakout sessions, attendees identified potential gaps, collaborative opportunities, and next steps to improve urban processes at global, regional, and microscales.

Why is the representation of cities important in ESMs? There is a vast literature examining the effect of aerosol emissions on climate, the biophysical effects of land-use and land-cover change on climate, the effects of building morphology on radiation, and the effect of urban heat islands on local temperatures. Also, the importance of scale in modeling urban systems 
is well established. What are less well known are the effects of urban systems on climate at different scales, the magnitude and linearity of feedbacks, biogeochemical feedbacks, and the future evolution of urban landscapes. For the effects of urban systems on climate at different scales, one challenge is to disentangle the signal from noise. The unknowns contribute to the debate as to whether representing urban systems in an ESM is a prerequisite for accurately representing climate. Do we need to represent urban systems to get global climate right? Furthermore, what does it mean to represent urban systems? Therefore, we asked the participants the question: Why is urban representation in an ESM important?

The discussion initially centered around whether or not urban systems need to be represented in a fully coupled ESM or whether ESMs should be linked to mesoscale and microscale models instead. The participants thought it was important to identify the goal of the ESM modeling exercise and the intended science question. Participants brainstormed on three potential aspects of urban and ESM relationships related to the inclusion of urban systems for better estimations of global climate, quantifying the impacts on human systems, and estimating the potential reduction in climate change.

- For studies focused on the global climate, urban systems are a source of emissions and a source of surface change. However, while emissions from urban systems are large, an explicit urban representation in an ESM may not be required to capture these effects. The emissions used in the CMIP simulations capture urban sources. For surface changes, there was a debate among participants as to whether the surface change was large or small.

- For studies focused on impacts on human systems, it was noted that a large fraction of the population lives in cities and wants to understand future climate change where they live. ESMs without an explicit urban representation may not accurately capture exposure to climate extremes such as heat waves. However, the CMIP6 effort to collect climate-related variables at the subgrid level may help, as several ESMs have urban tiles and will capture differences in temperature between urban and nonurban land types.

- For studies estimating the potential reduction in climate change, explicit representations of urban systems may be required. For example, if one was interested in quantifying the effects of 50\% more efficient buildings or a transition to white roofs on the local climate, then an urban system representation would be necessary for the ESM.

\section{Workshop findings}

Based on the keynote talks, panel discussions, and breakout groups, three distinct urban issues were identified that cut across the above workshop goals: (i) the urban footprint, (ii) hierarchy of models, and (iii) observation and sensing technologies.

The urban footprint. Urbanization and the growth of cities are entirely rearranging the global flow of resources and energy, localizing and concentrating use and consumption in a small part of the Earth's surface. While it is apparent that cities can influence the environment with a much larger footprint than their physical domains, and the effects from cities locally and regionally are clear, it is not so clear as to whether these effects are significant enough to affect the climate at global scales. Discussions identified the following gaps and opportunities in this aspect:

- Understand the control volume of the city: The ESM models fail to define the control volume (extent) of a city. Moreover, with respect to modeling, all urban processes are embedded in the first layer-a big problem! It is not ideal to consider urban areas and inhabitants as a very small component of the numerical representation. This is the likely reason for significant errors in capturing the planetary boundary layer heights over urban areas. 
- Urban heterogeneity: Urban heterogeneity is a key driver in urban systems. Currently, it is not well captured in models due to the lack of availability of ultra-high-resolution land-use datasets. A novel NUDAPT/WUDAPT (National Urban Database and Access Portal Tools/ World Urban Database and Access Portal Tools) approach can help with heterogeneity by digitizing urban information in three-major " $F$ " categories, i.e., form (geometry), fabric (material composition) content, and functionality (energy usage and waste products) (Ching et al. 2018).

- Scale-dependent urban parameterization: As we move from microscales to mesoscales to global scales, the effect of roughness elements in the urban canopy is muted. Therefore, the ESM community needs to design scale-dependent parameterizations to challenge current urban and nonurban parameterizations of physical processes under extreme weather cases, particularly boundary layer parameterizations under hyperconvective or extra-neutral conditions for a range of geographical locations. Cloud-aerosol interaction with urban environments needs further exploration with an emphasis on aerosol transport, cloud formation, and impacts on surface energy balance. This will also allow us to understand how the global climate is manifested when it interacts with urban environments.

The hierarchy of models. A hierarchy of models going from a detailed microscale representation of urban areas to global scales is needed to examine the effects of cities on our environment. There are already notable successes in modeling the urban atmosphere on a hierarchy of scales (Conry et al. 2015), and there are challenges in making improvements within and between those scales. Thus, maintaining and improving each element of the hierarchy will be essential.

Existing ESMs, which are especially used to understand global changes in climate, can already represent some aspects of urban processes, e.g., tiles in the NOAA Geophysical Fluid Dynamics Laboratory model for urban canyons and types of roofs that affect internal building temperature, wall/roof heat, heat fluxes, and water fluxes (Li et al. 2016). However, urban canopy parameterization has problems representing areas of very tall buildings. They typically do not extend outside the lowest layer of the atmosphere. The parameter values are not well defined for all cities globally, and the models may not be valid in extreme weather conditions. The heterogeneity of cities is also not well represented. Upscaling the urban effects through the planetary boundary layer is key to capturing the regional coupling but is poorly observed and modeled (Kristovich et al. 2019). Despite these limitations, they have been able to capture the basic properties of the urban heat island effects.

It should be possible to build better parameterizations of urban areas in ESMs using computational fluid dynamics (CFD) or microscale models that solve the fundamental fluid dynamics equations in an urban domain. However, a lack of validating data is a large obstacle to progress in using CFD models for the urban atmosphere. At the same time, for studies focused on the global climate, an explicit urban representation in an ESM may not be required to capture the effects of emissions, as they are parameterized, except to study the effects of urbanization.

Observations and sensing technologies. Emerging smart city sensing technologies offer a unique opportunity for use in model evaluation and development. There is an increase in the availability of data related to processes in urban centers, with the potential to deepen our understanding of urban-climate interactions. Some of these data are fueled by many "smart cities" projects. Applying analytics and optimization methods to this Big Data helps cities plan, manage, make better decisions, and strategize how to adapt to and mitigate climate change. - Data sources related to urban areas have rapidly increased in recent years with multiple 
technological advances. For instance, experimental infrastructure such as the Chicago's Array of Things (AoT) project provides air quality at a neighborhood scale at 30-s temporal resolution (Catlett et al. 2017). Similarly, networks such as Weather Underground provide neighborhood-scale meteorological measurements.

- Data from remote sensing devices (satellite-borne) are also increasing in both temporal and spatial resolution as well as quality.

- With the nearly universal use of mobile devices, cellular phone carriers and, increasingly, mobile phone hardware and application companies (e.g., Google, Apple, Twitter, and Facebook), have collected detailed data about human movement, from which these companies (and their partners) are building sophisticated models forecasting traffic patterns in urban areas (Nair et al. 2019). Such data can provide additional factors in modeling urban emissions from both vehicle traffic and buildings.

With the continuous influx of new types of data, thanks to cheap, lightweight sensors, GPS, social networks, wireless communications, and the like, the ability to effectively organize and analyze information is becoming increasingly challenging. Consequently, cities, and scientists plumbing data for studying urban processes and impacts, require new methods of collecting, synthesizing, filtering, and processing data from many independent sources, as well as tools for data analytics, visualization, and integrating with computational models.

\section{Recommendations}

At the end of the workshop, the participants agreed to focus on enhancing the predictability of the Earth system, including modeling its various components at finer and finer spatial scales that were not previously possible. Examples include pushing the boundaries of enhancing spatial resolution using adaptive meshes and more powerful computers, integrating remote sensing (satellite) observations, and investing in machine learning as a complement to high-resolution modeling for ESMs. Unique data assimilation capabilities should be used to improve climate models across spatiotemporal scales. Future urban ESMs should help model chemical, biological, and radiological (CBR) hazards in vulnerable complex urban hot spots. Hence, gaining an understanding of and developing predictive capabilities for flow and turbulence in a complex urban environment is of great importance for urban health and security. In addition, improvements in the urban process in ESMs will enhance sustainable urban development (Ando et al. 2019). This will provide seamless prediction capability across space-time scales, understanding of processes, Earth system components, types of observations, and data assimilation for user-oriented impact-based urban hydrometeorological forecasts. Overall, the workshop identified four priority areas for future research:

1) Cataloging data archives to develop model testbeds. Data are needed for assimilation, for parameter evaluation, and to develop new parameters. There is a need to create a central repository, so users can work with data that already exist and add new data as they are collected. For this purpose, we should use current cities that already have Big Data as modeling testbeds for evaluation.

2) Design field campaigns. There should be research investments to integrate in situ measurements and remote sensing and leverage alternative data sources to study urban multiscale processes. Urban ESMs should collaborate with stakeholders and community members to sustain data collection and learn about the future direction of the city. Field campaigns should go beyond the urban boundary to understand coupling across the rural-urban gradient.

3) Build networks. Networks should coordinate and organize long-term data collection across a variety of data sources. Networks can be used to communicate across a wide range of 
scales and to connect different interest groups, integrating synergistic activities to maximize data availability and flow.

4) Cross-scale model development. Models need to consider the unique properties of a city and heterogeneity at all scales. They should design idealized cities for different regions (e.g., the Army Research Laboratory's Meteorological Sensor Array research facility in New Mexico designs artificial urban environments to study idealized urban flows), but not neglect that cities change over time.

Based on the findings from the identified urban issues, the workshop participants produced following broad recommendations for future urban ESM research:

- There is an urgent need for increased interactions between those that work on urban issues across the scales from local to global, including the modeling of the processes relevant across these scales in the study of climate change.

- A need for an ongoing commitment and perhaps an international forum to maintain a continuous dialogue about these interactions across the wide range of science communities represented at the workshop and beyond.

- Due to relatively coarse spatial resolution and inadequacies in considering urban processes, policymakers should be warned that current ESMs should not be used directly for policy considerations at scales approaching that of the urban areas.

Acknowledgments. We thank all the participants for actively participating in the workshop. We thank Dr. Fei Chen (NCAR) and Dr. Lei Zhao (University of Illinois at Urbana-Champaign) for reading the summary manuscript and verifying the factual description of the workshop and accuracy of the content. This workshop was funded through interagency support from the U.S. Department of Energy, the Army Research Office, NASA, and NOAA.

\section{References}

Ando, A., and Coauthors, 2019: 2019 CURES Connections Workshop: New voices and paths to urban sustainability. NSF Sustainable Urban Systems Workshop Rep., 59 pp., https://dpi.uillinois.edu/UserFiles/Servers/Server_964145/File /CURES-2019-NSF-report-final.pdf.

Catlett, C. E., P. H. Beckman, R. Sankaran, and K. K. Galvin, 2017: Array of things: A scientific research instrument in the public way: Platform design and early lessons learned. Proc. Second Int. Workshop on Science of Smart City Operations and Platforms Engineering, Pittsburgh, PA, Association for Computing Machinery, 26-33, https://doi.org/10.1145/3063386.3063771.

Ching, J., and Coauthors, 2018: WUDAPT: An urban weather, climate, and environmental modeling infrastructure for the Anthropocene. Bull. Amer. Meteor. Soc., 99, 1907-1924, https://doi.org/10.1175/BAMS-D-16-0236.1.

Conry, P., A. Sharma, M. J. Potosnak, L. S. Leo, E. Bensman, J. J. Hellmann, and H. J. S. Fernando, 2015: Chicago's heat island and climate change: Bridging the scales via dynamical downscaling. J. Appl. Meteor. Climatol., 54, 14301448, https://doi.org/10.1175/JAMC-D-14-0241.1.
Fernando, H. J. S., 2010: Fluid dynamics of urban atmospheres in complex terrain. Annu. Rev. Fluid Mech., 42, 365-389, https://doi.org/10.1146/annurev -fluid-121108-145459.

Kristovich, D. A. R., E. Takle, G. S. Young, and A. Sharma, 2019: 100 years of progress in mesoscale planetary boundary layer meteorological research. A Century of Progress in Atmospheric and Related Sciences: Celebrating the American Meteorological Society Centennial, Meteor. Monogr., No. 59, Amer. Meteor. Soc., https://doi.org/10.1175/AMSMONOGRAPHS-D-18 $-0023.1$.

Li, D., S. Malyshev, and E. Shevliakova, 2016: Exploring historical and future urban climate in the Earth System Modeling framework: 1. Model development and evaluation. J. Adv. Model. Earth Syst., 8, 917-935, https://doi .org/10.1002/2015MS000578.

Nair, D. J., F. Gilles, S. Chand, N. Saxena, and V. Dixit, 2019: Correction: Characterizing multicity urban traffic conditions using crowdsourced data. PLOS ONE, 14, e0215728, https://doi.org/10.1371/journal.pone.0215728. 